\title{
Calculation of the Thermoacoustic Stability of a Main Stage Thrust Chamber Demonstrator
}

\author{
Alexander Chemnitz and Thomas Sattelmayer
}

\begin{abstract}
The stability behavior of a virtual thrust chamber demonstrator with low injection pressure loss is studied numerically. The approach relies on an eigenvalue analysis of the Linearized Euler Equations. An updated form of the stability prediction procedure is outlined, addressing mean flow and flame response calculations. The acoustics of the isolated oxidizer dome are discussed as well as the complete system incorporating dome and combustion chamber. The coupling between both components is realized via a scattering matrix representing the injectors. A flame transfer function is applied to determine the damping rates. Thereby it is found that the procedure for the extraction of the flame transfer function from the CFD solution has a significant impact on the stability predictions.
\end{abstract}

\section{Introduction}

High frequency combustion instabilities, i.e. the mutual amplification of acoustic oscillations and heat release fluctuations, are a recurring issue in rocket engine development. Current trends towards the use of alternate propellant combinations as well as the reduction of safety margins in favor of increased system efficiency pose additional challenges for the design of thermoacoustically stable engines. Several thrust chamber demonstrators (TCDs) featuring key elements of next generation rocket engines have been proposed by ArianeGroup [5]. Demonstrator TCD2 is designed with a low pressure loss between the oxidizer dome and the combustion chamber. While this reduces the power demands of the turbopumps, it increases the risk of acoustic coupling between chamber and injection system.

\footnotetext{
A. Chemnitz $(\varangle) \cdot$ T. Sattelmayer

Chair of Thermodynamics, Technical University of Munich, Boltzmannstr. 15,

85747 Garching, Germany

e-mail: chemnitz@td.mw.tum.de

T. Sattelmayer

e-mail: sattelmayer@td.mw.tum.de 
For the efficient assessment of the stability behavior a hybrid methodology [9] has been developed and is continuously improved. In the current study, this approach is used to analyze the TCD2 design regarding its thermoacoustic stability. The basic chamber acoustics have been characterized previously [2]. The pure first transverse mode $\left(T_{1}\right)$ has been found to possess the lowest damping capability of the modes of first transverse order. Thus, it has been selected for further investigation in the present study. Thereby the focus lies on the dynamics of the oxidizer dome and the influence of the flame response on the chamber stability.

After a short introduction of the test case, an overview of the current version of the stability assessment approach is given. The numerical setups used in the different steps of the procedure are outlined in the subsequent section. The acoustics of the isolated dome are studied before the coupled system as well as the impact of the flame response are discussed.

\section{Test Case}

The test case under consideration is the virtual Thrust Chamber Demonstrator TCD2. The geometry of the different components is shown in Fig. 1. The combustion chamber (Fig. 1a) has a diameter of $390 \mathrm{~mm}$ and a characteristic length of $800 \mathrm{~mm}$. Under operation the engine produces about $1 \mathrm{MN}$ of thrust with a nominal chamber pressure of $p_{c}=100$ bar. It is fed with liquid hydrogen and oxygen at a total mass flow rate of $\dot{m}_{t}=226.7 \mathrm{~kg} / \mathrm{s}$ and a mixture ratio of $O / F=6$. Oxygen is injected at a temperature of $T_{o x}=95 \mathrm{~K}$ and hydrogen at $T_{f}=110 \mathrm{~K}$.

The domes distribute the propellants from the feed system to the injector elements. The geometry of the oxidizer dome is shown in Fig. 1b. The grayed region around

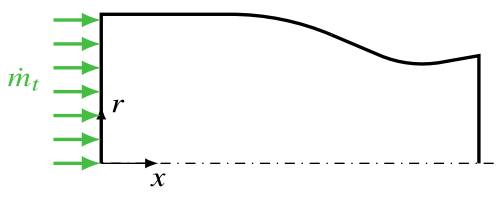

a

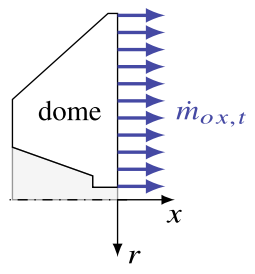

b

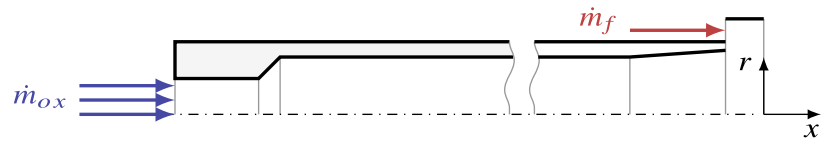

c

Fig. 1 a Combustion chamber. b $\mathrm{O}_{2}$ dome. $\mathbf{c}$ Injector 
the axis contains the igniter and is not part of the dome volume. In total 396 injection elements link the dome with the combustion chamber. Their geometry is shown in Fig. 1c. Each injector comprises a throttle on its upstream side to reduce acoustical coupling between the feed system and the chamber.

\section{Stability Assessment Procedure}

The stability assessment procedure is designed to evaluate the thermoacoustic stability of a rocket engine at reasonable computational cost and to allow for the design of stabilizing measures if necessary. The overall approach is sketched in Fig. 2. The key element is an eigenvalue analysis of the Linearized Euler Equations. The complex eigenvalue consists of the oscillation frequency (real part) and a damping rate (imaginary part). The latter characterizes the modal stability behavior of the chamber with a positive damping rate corresponding to a stable mode. The eigenvalue analysis involves several sub-models. First, the mean flow is required, which is the reference state for the perturbation equations. Second, a Flame Transfer Function (FTF) represents the flame response. The different components of the procedure are discussed in the following.

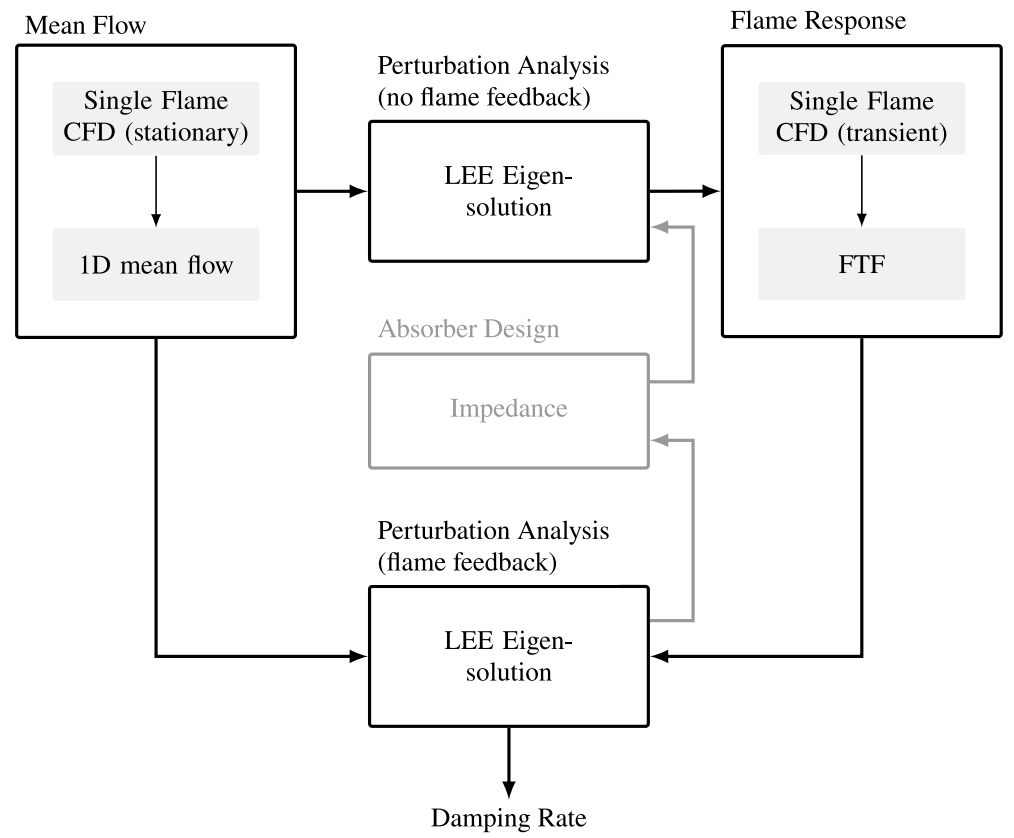

Fig. 2 Schematic of the stability assessment procedure 


\subsection{Perturbation Analysis}

In the perturbation analysis eigensolutions of the Linearized Euler Equations in frequency space are computed. The eigenvalue is composed of the oscillation frequency $\omega$ and the damping rate $\alpha$ while the eigenvectors correspond to the complex oscillation amplitude distribution of the perturbed variables. To reduce computational cost, the circumferential $(\theta)$ distribution of the complex amplitudes $\left({ }^{\wedge}\right)$ is accounted for [6] by the analytic ansatz

$$
\hat{\phi}=\tilde{\phi} \exp (i n \theta)
$$

with $n$ the transverse order of the mode under consideration, where $n=0$ corresponds to a non-transverse mode. This allows to compute transverse modes on a two-dimensional domain. Besides the linear mass and momentum equations, a pressure equation derived from energy conservation is solved:

$$
i(\omega+i \alpha) \hat{p}+\overline{\mathbf{u}} \cdot \nabla \hat{p}+\hat{\mathbf{u}} \cdot \nabla \bar{p}+\kappa(\bar{p} \nabla \cdot \hat{\mathbf{u}}+\hat{p} \nabla \cdot \overline{\mathbf{u}})-\frac{1}{\kappa-1}(\hat{\mathbf{u}} \bar{p}+\overline{\mathbf{u}} \hat{p}) \cdot \nabla \kappa=(\kappa-1) \hat{\dot{q}}
$$

Here, $p$ denotes the pressure, $u$ the velocity, $\kappa$ is the isentropic coefficient and $\dot{q}$ the volumetric heat release. The term on the right hand side represents the fluctuation energy source due to heat release oscillations from the flame response.

Two perturbation analyses are carried out in the stability assessment procedure. First, without heat release oscillations in order to get the pressure amplitude distribution for the characterization of the flame response. Second, for the final determination of the chamber's stability behavior.

\subsection{Mean Flow}

The mean flow is the reference state for the perturbation analysis. Aside from the acceleration in the nozzle, the flow in the chamber is dominated by a high number of diffusion flames. To avoid the excessive computational effort required to resolve the associated small-scale structures, a quasi-one-dimensional equivalent flow field is used [9]. It is based on radially averaged profiles from single flame simulations with the heat release due to combustion being represented by an axial energy source term distribution. The effect of neglecting radial gradients has been studied by Chemnitz et al. [3]. The calculation procedure for the mean flow has been enhanced to obtain flow fields that are fully consistent with the Euler Equations while reproducing axial profiles of sound speed and isentropic compressibility, as described in the following.

The computational domain of the mean flow covers the whole combustion chamber. It is designed to reproduce the radially averaged (index ${ }_{1 D}$ ) heat-release distribution as well as the sound speed $c$ and isentropic compressibility $\eta_{s}$ of a single flame. In the cylindrical chamber section, a target density profile is calculated using 


$$
\rho_{1 D}=\frac{1}{\eta_{1 D} c_{1 D}^{2}} .
$$

The non-linear Euler Equations are solved with the gas-constant computed as

$$
R=\frac{p}{\rho_{1 D} T}
$$

In a post-processing step the desired sound speed distribution is obtained by adapting the isentropic coefficient

$$
\kappa=c_{1 D}^{2} \frac{\rho}{p}
$$

The temperature dependence of the enthalpy is modeled based on the local flow composition in the single flame simulation. Towards the nozzle throat, the isentropic coefficient is blended to the value of the CFD-solution in order to ensure correct localization of the sonic line.

Due to the compact engine design combustion still occurs in the convergent part of the nozzle. In a previous study [2] a correction has been proposed to account for the influence of the flow acceleration in the nozzle, which is not included in the single flame simulation. The heat release is transformed from its spatial dependence to a function of the combustion progress, which is represented by the heat released upstream and corrected for the flow velocity:

$$
\dot{q}(x)=\frac{1}{A_{x}} \dot{q}_{s f}\left(\int_{x_{0}}^{x} \dot{q} A_{x} d \check{x}\right) \frac{u_{s f}}{u}
$$

with $_{s f}$ denoting the single flame solution, $x_{0}$ is the location of the entrance to the convergent nozzle section and $A_{x}$ the local crossectional area. By normalizing the single flame and the nozzle velocity profiles with their respective values at $x_{0}$, steadiness of the heat release profile is ensured. In the original work [2], a non-iterative correction of the heat release was applied: A mean flow simulation without heat-release in the nozzle was conducted and the one-dimensional axial velocity profile extracted. The heat release was then corrected and a second mean flow simulation performed. This process has been changed to solving the differential equations for one-dimensional compressible flow with isentropic heat addition and area change in a pre-processing step. This on the one hand eliminates the need for the first CFD simulation and on the other hand ensures convergence of the corrected heat release. The axial distribution of the species that describe the temperature dependence of the enthalpy is corrected consistently. 


\subsection{Flame Response}

To include the flame response in the stability analysis, the oscillating heat release due to the perturbations in the chamber is required (cf. Eq. 2). The dominant contribution has been found to come from the coupling between heat release and pressure oscillations [9], represented via a transfer function

$$
\hat{\dot{q}}=F T F \hat{p}
$$

It is extracted from a transient single flame simulation. Thereby source terms account for the effect of the overall chamber acoustics on the flow in the single flame domain.

To characterize the pressure coupling FTF of a transverse mode, a flame located at the pressure anti-node is considered. Based on pressure mode shape and eigenfrequency of an eigenvalue analysis without flame feedback, source terms are derived. However, the axial distribution of the pressure amplitude in the chamber is governed by its cut-off-behavior [9]. Since the diameter of the single flame domain is significantly smaller than that of the combustion chamber, no transverse mode occurs at the frequency of interest. Instead, longitudinal acoustics of the single flame domain interfere with the perturbations induced by the source terms. Several studies have been performed on how to excite the single flame. Schmid [7, 8] proposed an approach that estimates the acoustic mass flow at the domain boundaries based on the analytical solution of uniform duct-flow acoustics. Schulze [9] replaced the analytical axial pressure amplitude distribution by that obtained from the eigenvalue study of the combustor. However, in the presence of mean flow, deviations between the targeted and resulting pressure fluctuations were found [8]. Thus, the actual pressure fluctuations occurring in the single flame simulation (index ${ }_{c}$ ) differ from those that are used for the calculation of the source terms (index ${ }_{e x}$ ). Conclusively there are two possible pressure amplitude profiles that can be used for the extraction of the FTF. Both approaches seem reasonable: As the source terms are designed to mimic the impact of a prescribed pressure amplitude distribution on the flame, corresponding heat release fluctuations need to take this distribution as reference. However, the actual pressure fluctuations that occur in the single flame simulation lead to a heat release response as well. The consequences of the choice of the respective reference pressure amplitude distributions will be addressed in Sect. 5.3.

To improve the accuracy of the model for the acoustics' effect on the flame, the injection of mass at the domain boundaries has been replaced by volumetric source terms across the whole single flame domain. It has been found that the shape of the acoustic pressure distribution is not influenced by the presence of small scale diffusion flame structures in the mean flow [2]. Thus, the superimposed pressure can be assumed to be independent of the radial position in the flame. Neglecting convective effects of the mean flow, the mass source corresponding to an acoustic pressure oscillation is obtained as

$$
\dot{\mathcal{S}}_{m, a}=\frac{1}{c^{2}} \frac{\partial p_{a}^{\prime}}{\partial t} .
$$


If this equation is evaluated integrally for a whole chamber cross-section, it can be transformed into the form used by Schmid and Schulze, showing consistency of the approaches. From the radial gradient of the volumetric source term distribution, the radial acoustic mass flow is obtained:

$$
-d \dot{m}_{r, a}=\dot{\mathcal{S}}_{m, a} d V
$$

Equation 9 can be numerically integrated in radial direction with the boundary condition $\dot{m}_{r, a}=0$ at the domain axis. With this mass flow, the convective transport of flow variables can be evaluated at each location and the associated volumetric source term for a variable $\phi$ is obtained as

$$
\dot{\mathcal{S}}_{\phi}=\frac{1}{A_{r}} \frac{\partial}{\partial r}\left(\dot{m}_{r, a} \phi d A_{r}\right)
$$

\subsection{External Components and Design Adaption}

External components can be coupled via scattering matrices or modeled as impedance boundary conditions. In the present study a scattering matrix is used to model the injectors that connect the chamber to the oxidizer dome, following the approach of Schulze [9].

The use of an impedance boundary condition is particularly advantageous for the incorporation of damping devices. In the case of a combustion instability, the application of dampers is a common approach to adapt the chamber acoustics. Chemnitz et al. [1] showed how a systematic variation of the absorber impedance generates characteristic surfaces in the three-dimensional impedance-frequency and impedancedamping rate spaces. These surfaces are evaluated following the grayed out path in Fig. 2 and provide constraints for the absorber characteristics. The detailed absorber geometry can then be designed based on these constraints, without the necessity to explicitly include the chamber acoustics in the associated calculations.

\section{Numerical Setup}

Each of the stabilization procedure's components (Fig. 2) requires specific numerical simulations. While the calculation of the mean flow is outlined in Sect. 3.2, additional information on the setups for the single flame and the perturbation simulations are given in the following. 


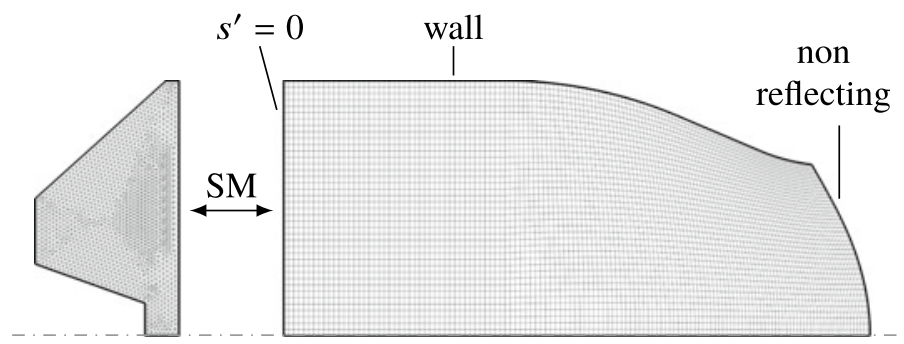

Fig. 3 Computational domain of the perturbation simulation

\subsection{Eigensolution Study}

The domain for the perturbation analysis is two-dimensional axis-symmetric in the $r-z$-plane. It is shown along with the used mesh and boundary conditions in Fig. 3. In addition to the coupling with the dome, entropy fluctuations $\left(s^{\prime}\right)$ are set to zero at the upstream boundary of the chamber. The remaining dome boundaries are modeled as walls. The eigensolution study is performed with slightly different approaches in chamber and dome.

In the chamber, the non-isentropic Linearized Euler Equations are solved. They are discretized with quadratic finite elements and stabilization is added to suppress numerical instabilities. The strength of the stabilization is controlled via the parameter $\tau_{s}$. Its value is selected based on the consistency of the results with basic flow-field properties that are expected from analytical solutions. The domain covers the flow up to slightly supersonic conditions. That way, the acoustic boundary condition imposed by the supersonic flow is retained.

In the dome, the Helmholtz Equations are solved, discretized with linear finite elements.

\subsection{Single Flame}

The single flame simulations are conducted as Reynolds Averaged Navier Stokes (RANS) simulations. The k- $\epsilon$-model is used for turbulence closure following the results of a study by Chemnitz et al. [4]. Combustion is modeled via an isobaric, diabatic equilibrium chemistry model with a $\beta$-pdf of the mixture fraction to account for turbulence-chemistry-interaction. The turbulent Schmidt number is calibrated for the flame-length to match the predictions of ArianeGroup.

The simulations are conducted with ANSYS® Fluent 18.0 with the domain and boundary conditions shown in Fig. 4. It is dimensioned to preserve the area ratio between injector and chamber cross section. The mesh consists of about 266000 cells 
Fig. 4 Computational domain for single flame simulations ([2], adapted)

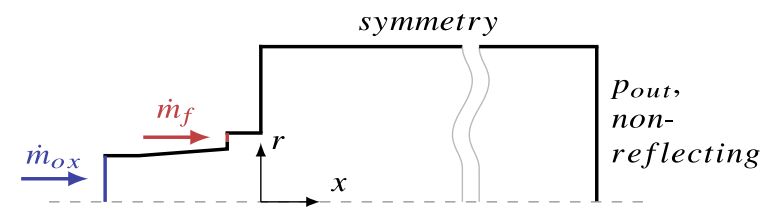

with about 1400 cells in axial and 190 in radial direction. The high axial resolution is required for the gradient-based extraction of the one-dimensional heat release distribution.

\section{Results}

Based on the methodology and setups outlined in the previous sections, the stability analysis is conducted. In the following, the acoustics of the isolated dome are discussed before considering the coupled system. Finally, the impact of the flame response on the stability behavior is addressed. The mean flow has already been presented in a previous publication [2].

\subsection{Dome Acoustics}

To acoustically characterize the dome independent of the chamber, an eigenvalue analysis for modes of transverse order $n=0$ and $n=1$ is conducted. All non-axis boundaries are treated as walls, since the total area of the injectors holes amounts to less than $4 \%$ of the dome base plate area.

The shapes of the first two non-transverse dome modes are shown in Fig. 5. Due to the conical dome geometry there is no clear distinction between longitudinal and radial modes. Thus the notation $C$ is used to denote any non-transverse mode type.

The eigenfrequencies of the dome (superscript ${ }^{D}$ ) are plotted in Fig. 6 along with that of the chamber $T_{1}$ (superscript ${ }^{C}$ ). In the dome the first transverse mode occurs at frequencies below that of any non-transverse mode. This can be attributed to the

Fig. 5 Normalized dome pressure amplitude distribution (zeroth transverse order)
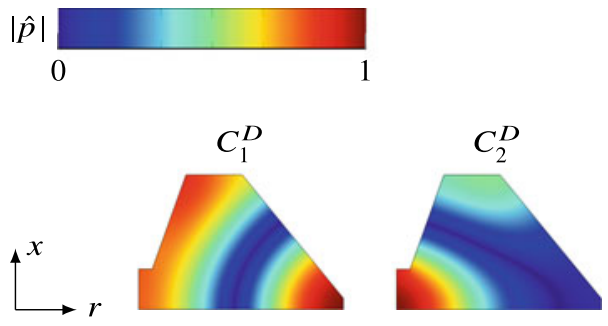
Fig. 6 Dome eigenfrequencies

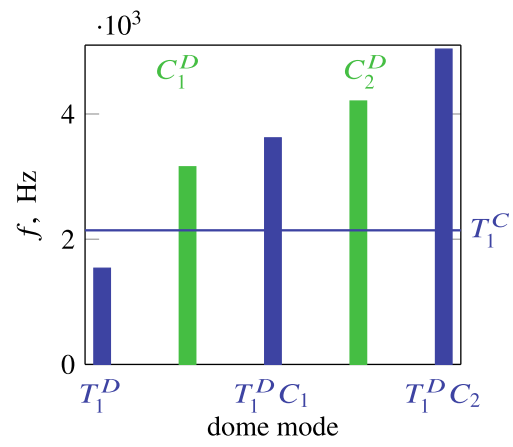

dome's low length to diameter ratio. The $T_{1}^{D}$ mode is closest to the chamber $T_{1}^{C}$, followed by the $C_{1}^{D}$. The subsequent dome modes' frequencies are rather equidistant.

The shapes of the modes of first transverse order are shown in Fig. 7. For the pure $T_{1}^{D}$ mode the amplitude distribution resembles that of a cylindrical duct. However, for the subsequent two combined modes again there is no clear distinction between radial and longitudinal structures.

\subsection{Coupled Acoustics}

By coupling the dome volume to the chamber via a scattering matrix, the eigenmodes of the full system can be obtained. The frequency of the coupled $T_{1}$ mode is nearly identical to that of the chamber (Fig. 6). The pressure amplitude distribution of the $T_{1}$ mode in the coupled domain is shown in Fig. 8. The mode shape of the chamber
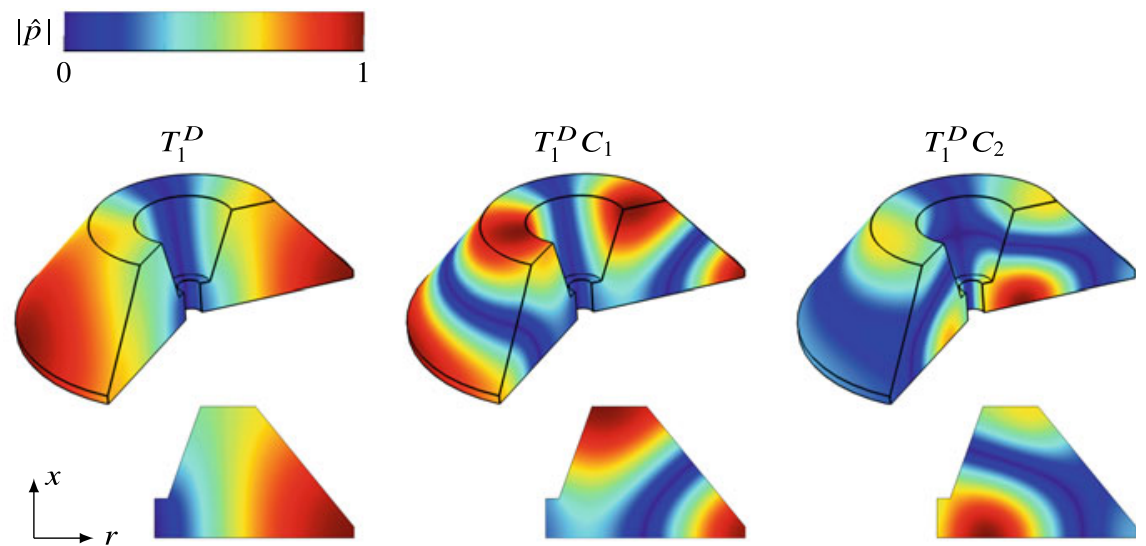

Fig. 7 Normalized dome pressure amplitude distribution (first transverse order) 

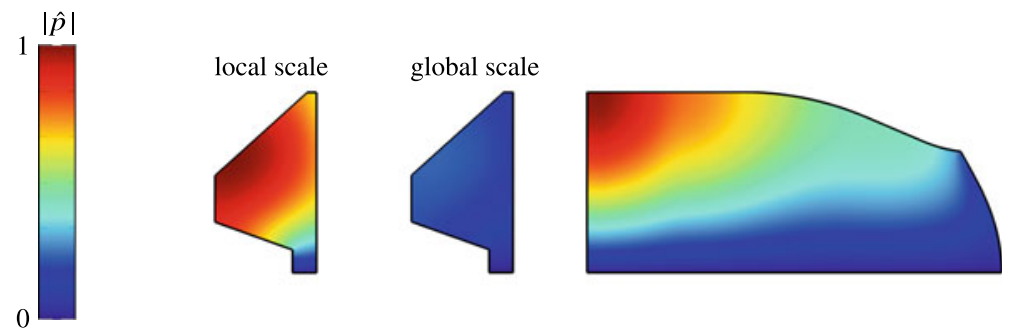

Fig. 8 Normalized pressure amplitude distribution in the coupled domain

is found to show no significant change compared to the case without dome [2]. In the dome itself, only weak pressure disturbances are observed relative to the chamber. The shape of the pressure distribution in the dome (Fig. 8) shows a significant change compared to the previously discussed eigensolutions (Sect. 5.1). It takes the form of the radial pressure amplitude distribution at the chamber inlet. This behavior can be explained by the relative position of eigenfrequencies (Fig. 6). Despite the dome $T_{1}$ being closest to that of the chamber, they are separated by a difference of $\Delta f_{T_{1}} \approx 600 \mathrm{~Hz}$. Since the eigensolution of interest in the coupled system is that governed by the chamber acoustics, the dome shows only a weak acoustic response. However, close to the dome's $T_{1}$ eigenfrequency an eigenmode of the coupled domain exists as well. There the dome pressure distribution resembles that discussed in the previous section and significantly lower amplitudes occur in the chamber.
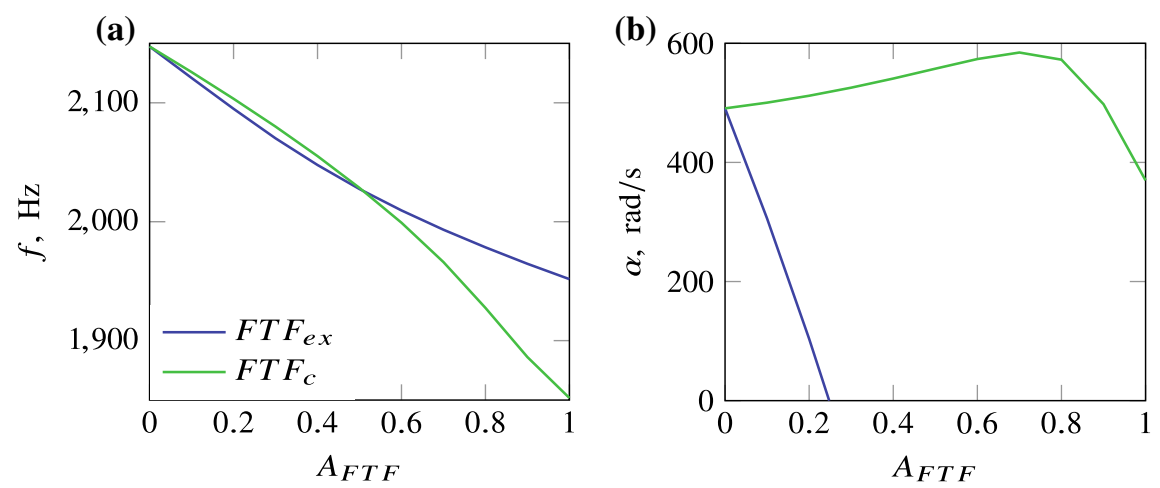

Fig. 9 (a) Eigenfrequency via FTF scaling (b) Damping rate via FTF scaling 


\subsection{Stability Behavior}

To assess the thrust chamber's stability behavior, the flame response is included in Eq. 2. A significant impact of the choice of the pressure amplitude for the FTF calculation on the predicted stability is observed. As outlined in Sect. 3.3, there is a deviation between the prescribed $(e x)$ and obtained $(c)$ pressure fluctuations that leads to different possible FTFs. To visualize the qualitatively different effect of the FTFs on the chamber stability, their amplitudes are scaled by a factor $A_{F T F}$ which is ramped from $A_{F T F}=0$ (no flame feedback) to $A_{F T F}=1$ (full flame feedback). The $T_{1}$ eigenfrequency via the FTF scaling is shown in Fig. 9a. The eigenfrequency decreases for both FTFs with a nearly identical behavior up to $A_{F T F} \approx 0.6$. From there on the frequency decrease for $F T F_{c}$ steepens. The most signifcant difference is observed for the damping rate, shown in Fig. 9b. While $F T F_{e x}$ exerts a strong destabilizing effect on the chamber, with $F T F_{c}$ stable operation is predicted. For low FTF scalings even a stabilizing effect on the chamber acoustics is observed. From $A_{F T F} \approx 0.6$, the damping rate starts to decrease but remains in the stable regime.

\section{Conclusions}

A stability assessment has been conducted for a virtual thrust chamber demonstrator via a hybrid approach. An adapted version of the basic methodology in terms of mean flow generation and flame excitation has been applied. The acoustics of the oxidizer dome as well as the coupled system of dome and chamber have been characterized. Finally, the impact of the flame response on the stability has been addressed.

Of the dome eigenmodes the $T_{1}$ is closest in eigenfrequency to the chamber $T_{1}$. Nevertheless, due to the still significant frequency distance only weak pressure fluctuations are present in the dome at the $T_{1}$ mode of the coupled system.

The extraction procedure for the flame transfer function showed a significant impact on the stability prediction. The acoustics of the single flame domain interfere with the modeled impact of the chamber acoustics on the flame. Thus, the obtained pressure fluctuations deviate from those used for the derivation of the excitation source terms. Depending of whether the prescribed or the observed pressure fluctuations are used for the FTF calculation, different stability predictions are obtained.

Regarding the flame transfer functions, an additional adaption of the excitation procedure is necessary to improve the agreement of prescribed and obtained pressure fluctuations. Aside from that, the current state of the stability assessment procedure provides a consistent way to perform efficient stability predictions and design adaptions for large scale rocket thrust chambers.

Acknowledgements Financial support has been provided by the German Research Foundation (Deutsche Forschungsgemeinschaft - DFG) in the framework of the Sonderforschungsbereich Transregio 40. 


\section{References}

1. Chemnitz, A., Kings, N., Sattelmayer, T.: Modification of eigenmodes in a cold-flow rocket combustion chamber by acoustic resonators. J. Propuls. Power (2019)

2. Chemnitz, A., Sattelmayer, T.: Acoustic characterization of virtual thrust chamber demonstrators. Annual Report SFB/TRR40, Chair of Thermodynamics, TUM (2018)

3. Chemnitz, A., Sattelmayer, T.: Influence of radial stratification on eigenfrequency computations in rocket combustion chambers. In: 8th EUCASS (2019)

4. Chemnitz, A., Sattelmayer, T., Roth, C., et al.: Numerical investigation of reacting flow in a methane rocket combustor: turbulence modeling. J. Propuls. Power 34(4), 864-877 (2017)

5. Eiringhaus, D., Riedmann, H., Knab, O.: Demonstratorbeschreibung TCD2 - v1.0 (2017)

6. Mensah, G.A., Moeck, J.P.: Efficient computation of thermoacoustic modes in annular combustion chambers based on bloch-wave theory. In: ASME Turbo Expo, vol. 4B (2015)

7. Sattelmayer, T., Schmid, M., Schulze, M.: Interaction of combustion with transverse velocity fluctuations in liquid rocket engines. J. Propuls. Power 31(4), 1137-1147 (2015)

8. Schmid, M.: Thermoakustische Kopplungsmechanismen in Flüssigraketentriebwerken. Ph.D. thesis, Technische Universität München (2014)

9. Schulze, M., Sattelmayer, T.: Linear stability assessment of a cryogenic rocket engine. Int. J. Spray Combust. Dyn. (2017)

Open Access This chapter is licensed under the terms of the Creative Commons Attribution 4.0 International License (http://creativecommons.org/licenses/by/4.0/), which permits use, sharing, adaptation, distribution and reproduction in any medium or format, as long as you give appropriate credit to the original author(s) and the source, provide a link to the Creative Commons license and indicate if changes were made.

The images or other third party material in this chapter are included in the chapter's Creative Commons license, unless indicated otherwise in a credit line to the material. If material is not included in the chapter's Creative Commons license and your intended use is not permitted by statutory regulation or exceeds the permitted use, you will need to obtain permission directly from the copyright holder.

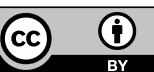

